\title{
Simultaneous EEG-fMRI in Human Epilepsy
}

\author{
Cameron JB Cunningham, Mohammed El-Fateh Zaamout, Bradley Goodyear, \\ Paolo Federico
}

\begin{abstract}
Electroencephalography (EEG) has been used to study and characterize epilepsy for decades, but has a limited ability to localize epileptiform activity to a specific brain region. With recent technological advances, high-quality EEG can now be recorded during functional magnetic resonance imaging (fMRI), which characterizes brain activity through local changes in blood oxygenation. By combining these techniques, the specific timing of interictal events can be identified on the EEG at millisecond resolution and spatially localized with fMRI at millimeter resolution. As a result, simultaneous EEG-fMRI provides the opportunity to better investigate the spatiotemporal mechanisms of the generation of epileptiform activity in the brain. This article discusses the technical considerations and their solutions for recording simultaneous EEG-fMRI and the results of studies to date. It also addresses the application of EEG-fMRI to epilepsy in humans, including clinical applications and ongoing challenges.
\end{abstract}

RÉSUMÉ: ÉEG-IRMf simultanés dans l'épilepsie chez l'humain. L'électroencéphalographie (ÉEG) est utilisée depuis des décennies pour étudier et caractériser l'épilepsie. Cependant son utilité pour localiser l'activité épileptique à une région spécifique du cerveau est limitée. Grâce aux progrès technologiques récents, un ÉEG de haute qualité peut maintenant être enregistré pendant l'imagerie par résonance magnétique fonctionnelle (IRMf), ce qui permet de caractériser l'activité cérébrale au moyen de changements locaux dans l'oxygénation du sang. En combinant ces techniques, la chronologie des événements intercritiques peut être précisée avec l'ÉEG à une résolution de millisecondes et localisée dans l'espace avec l'IRMf à une résolution millimétrique. L'ÉEG-IRMf peut donc fournir une meilleure évaluation des mécanismes tempo-spatiaux de la genèse de l'activité épileptiforme dans le cerveau. Dans cet article, nous discutons des problèmes techniques rencontrés dans l'enregistrement simultané de l'ÉEG-IRMf et de leur solution, ainsi que des résultats des études publiées jusqu'à maintenant. Nous discutons également de l'utilisation de l'ÉEG-IMRf dans l'épilepsie chez l'humain et particulièrement des applications cliniques et des défis à relever.

Can. J. Neurol. Sci. 2008; 35: 420-435

Electroencephalography (EEG) is a well established clinical and research tool for the characterization and localization of seizure disorders. However, traditional EEG recordings are limited in their ability to spatially localize epileptiform discharges, largely because the mathematical models used to determine the anatomical source of the discharges recorded from scalp electrodes can have multiple solutions (the 'inverse problem').

Hence, there continues to be research and development into improving EEG technology and algorithms to provide more unique solutions, as well as alternative, yet complementary, approaches to identifying the source of epileptiform activity by investigating metabolic activity. Indeed, there is a large body of evidence which indicates hemodynamic and metabolic changes are associated with epileptiform activity. In 1934, Gibbs et al ${ }^{1}$ used intracarotid temperature measurements to demonstrate an increase in cerebral blood flow during seizures compared to baseline in humans. Autoradiological approaches using $\left[{ }^{14} \mathrm{C}\right]$

From the Departments of Clinical Neurosciences (CC, MZ, BG, PF) and Radiology (BG, PF); The Hotchkiss Brain Institute (CC, MZ, BG, PF), University of Calgary; The Seaman Family MR Research Centre (CC, MZ, BG, PF), Calgary, Alberta, Canada.

Received December 7, 2007. Final Revisions Submitted April 11, 2008. Correspondence to: Paolo Federico, Department of Clinical Neurosciences, University of Calgary, Room C1214A, Foothills Medical Centre, 1403 29th Street NW, Calgary, Alberta, T2N 2T9, Canada. 
deoxyglucose have also shown an increase in glucose metabolism of up to three times baseline values during focal penicillin-induced seizures in rats. ${ }^{2}$ Using the same animal model, it has been shown that metabolic changes can extend away from the cortical focus to include other distal cortical and subcortical regions. ${ }^{3}$ As a result, brain imaging techniques such as single photon emission computed tomography (SPECT) and positron emission tomography (PET) are often used in clinical evaluations in epileptic patients to improve localization of the seizure focus by measuring perfusion and metabolism, respectively, during both ictal and interictal periods. ${ }^{4}$ Interictal SPECT shows cerebral hypoperfusion at the seizure focus in 40$60 \%$ of patients and ictal SPECT shows hyperperfusion at the seizure focus in $70 \%$ of patients. ${ }^{5-7}$ Interictal PET shows hypometabolism in $45-90 \%$ of all patients. This variability is dependant on the seizure focus (temporal versus extratemporal), epilepsy centre, and scanner used. ${ }^{4-6}$

In recent years, there has been increasing interest in using non-invasive imaging techniques such as functional magnetic resonance imaging (fMRI) in an effort to better anatomically localize interictal discharges and seizure foci. ${ }^{8-11}$ In a typical experimental setting, fMRI is used to detect changes in neuronal activity in response to tasks (e.g., cognitive and motor) or stimuli (e.g., visual and sensory). The most commonly used fMRI technique detects changes in venous deoxyhemoglobin content associated with increases in neuronal activity, the so-called Blood Oxygenation Level Dependent (BOLD) contrast technique. ${ }^{12}$ Deoxygenated hemoglobin within the red blood cells generates magnetic field inhomogeneities that are experienced by the nearby hydrogen nuclei of water molecules.

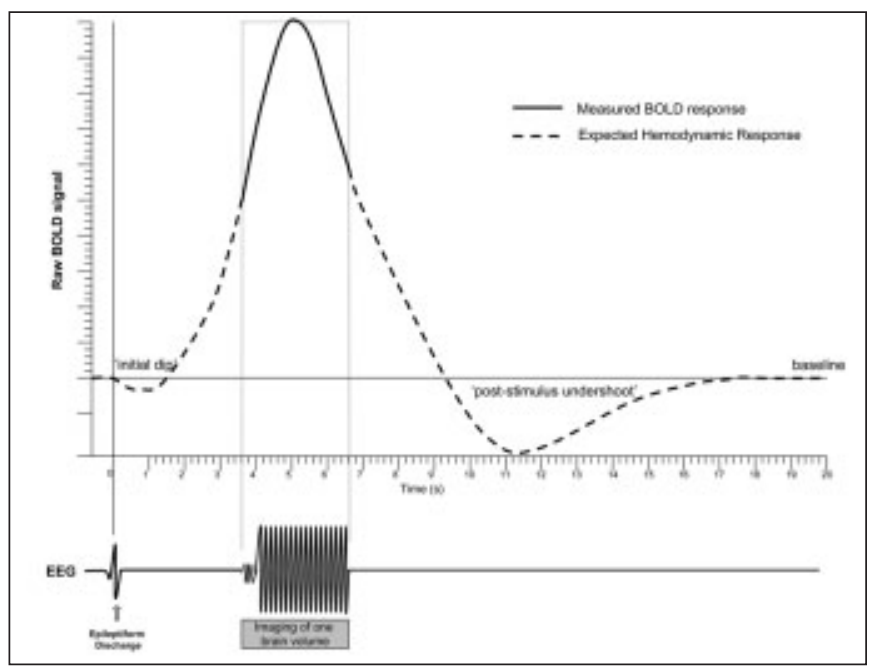

Figure 1: Spike-Triggered Hemodynamic Response Function (HRF) showing the measured and statistically modeled MR signal changes, based on a multi-phasic HRF. Theoretical data shows an increase in MR $B O L D$ signal following a measured interictal discharge on EEG, as well as an 'initial dip' in BOLD signal immediately following a discharge and a 'post-stimulus undershoot'after the response peak. EEG displays large artifact during MR image collection.
In response to a regional increase in neuronal activity, there is an increase in cerebral blood flow and cerebral blood volume that exceeds the rate of oxygen extraction from the blood, resulting in a decrease in the local deoxygenated hemoglobin concentration. These events reduce local magnetic field inhomogeneities, and ultimately increase MR image voxel (a three-dimensional volume element) intensity when the brain is imaged using specific MR sequences (e.g., gradient echo imaging). Currently, it is believed that the magnitude of the BOLD signal reflects the synaptic input and intracortical processing of a given volume (i.e., its total/overall synaptic activity) rather than the spiking output of the neurons in that area, ${ }^{13}$ and hence the increase in synaptic activity accompanying epileptiform activity is believed to give rise to a BOLD signal.

The temporal evolution of BOLD signal changes is in the form of a characteristic curve called the hemodynamic response function, or HRF [see ${ }^{14,15}$ for summary]. An HRF models the typical change in BOLD signal over time following a stimulus or an epileptiform discharge measured with EEG (Figure 1). Immediately following a stimulus, the BOLD signal and the oxyhemoglobin concentration have been shown in some studies to decrease briefly, often called the 'initial dip', which is thought to reflect an increase in oxygen metabolism and oxygen extraction before blood flow increases to the area. ${ }^{16-18}$ Following the initial dip is the characteristic increase in cerebral blood flow and cerebral blood volume that gives rise to an increase in BOLD signal, which returns slowly to baseline over several seconds, potentially dipping temporarily below baseline (called the poststimulus undershoot). ${ }^{18,19}$

The success of fMRI studies of epilepsy has partially driven the development of EEG technology that is compatible with the magnetic field environment to permit scalp EEG recordings during fMRI experiments. The combination of EEG and fMRI exploits the strengths of each modality in order to potentially obtain more precise temporal and spatial information about epileptogenic regions: EEG would provide high temporal resolution for precise timing of epileptiform activity and fMRI would provide high spatial resolution for precise spatial localization of epileptiform activity.

The technology required to record high-quality EEG in the electromagnetically noisy environment of the MR scanner has been improving steadily since EEG and fMRI were first combined in $1993 .{ }^{20}$ At that time, it was possible to record good quality EEG only in a static MR environment (i.e., during periods when MR images were not being acquired). More recently, techniques to remove the electrical noise introduced into EEG recordings as a result of the repeatedly changing magnetic fields used during MR image acquisition have been developed, thus permitting the simultaneous acquisition of EEG and MR images. ${ }^{21}$ Using these approaches, EEG-fMRI has been used successfully in cognitive and sleep studies [see ${ }^{22}$ for example], and it has been used widely in studies of epilepsy [see 9,23-25 for examples].

In this review, we discuss the rationale for combining EEG and fMRI in human epilepsy research, highlighting the main technical issues that arise from combining the two techniques and their solutions, as well as review results from key studies and discuss future directions in research [for a more detailed review of animal fMRI studies of epilepsy, see Blumenfeld ${ }^{26}$ and Mirsattari et $\mathrm{al}^{27}$. 


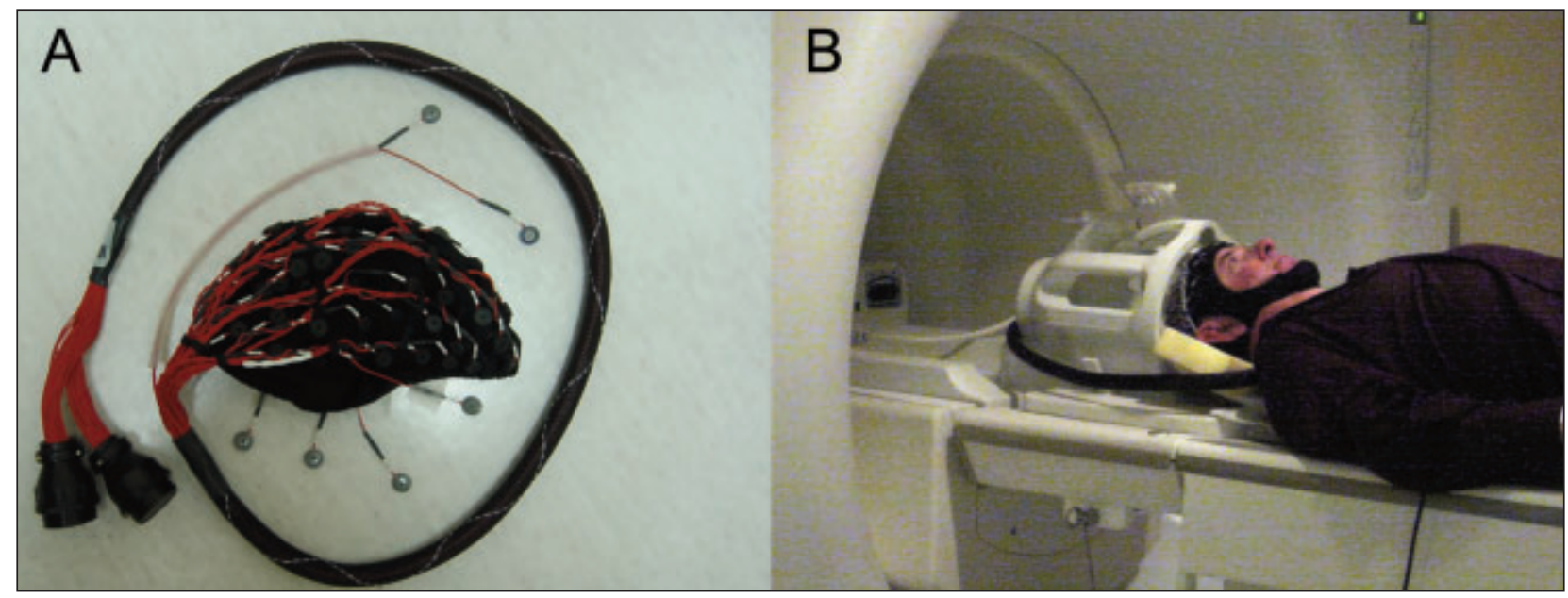

Figure 2: A. 64 channel MRI-compatible EEG cap (Compumedics Neuroscan) B. Patient being prepared for a EEG-fMRI recording.

\section{Methodological Considerations and Developments MR safety}

Functional MRI is considered to be a safe and non-invasive means to measure brain activation, as it does not produce ionizing radiation; however, certain factors must be considered to ensure patient safety when combining EEG with fMRI. The MR safety concerns arise from the different types of electromagnetic energy applied, namely, static magnetic fields, changing gradient fields, and radiofrequency pulses, which will be discussed below.

Although epidemiological evidence of the long-term effects of static magnetic fields is insufficient to suggest any health detriment, ${ }^{28}$ static magnetic fields have been shown to influence living cells in culture after long-term exposure, potentially warranting further investigation of health risks [ ${ }^{29,30}$ for review of patient safety in MR]. Additionally, with the use of increasing magnetic field strengths (greater than 3 Tesla), heightened caution must be exercised, especially in patients with implanted medical devices or pregnant women. ${ }^{31}$ Strong magnetic fields can cause ferromagnetic materials such as iron, cobalt, and nickel to become projectiles; thus, these must be removed from the MR environment. Furthermore, the movement of a conducting loop in a static magnetic field can induce current in that loop, especially if it has low impedance. ${ }^{32}$ While EEG recording assemblies typically have high impedances and current limiting resistors to prevent induced current, care must be taken to prevent the formation of low-impedance loops through bridging of leads or contact of wires with the subject. ${ }^{32}$ Heating at the contact points between the electrodes and scalp could result from eddy currents within the electrode and/or electrode paste, or an antenna effect, where a maximal electric field is produced at the tip of a device. ${ }^{32}$ Further caution is also necessary when scanning patients who may have devices such as guide wires, catheters, and other electrodes present which may result in heating due to the antenna effect. ${ }^{33}$ These precautionary steps have been widely implemented. In all studies performed at our centre, no problems due to electrode heating have occurred and safety tests performed in our lab show minimal temperature increases.

Radiofrequency pulses and changing gradient fields can also generate current in low impedance loops that could result in electric stimulation (below $100 \mathrm{kHz}$ ) or heating (above 100 $\mathrm{kHz}){ }^{32}$ Temperature increases occurring during scanning are difficult to measure, so exposure to radiofrequency pulses are quantified by the 'specific energy absorption rate', or SAR, defined as the average energy dissipated in the body per unit of mass and time. The MR parameters in routine use typically do not produce dangerous SAR levels; however, SAR has been shown to increase in the presence of EEG electrodes and high magnetic field strengths. Hence, SAR needs to be closely monitored with each EGG-fMRI study. ${ }^{34}$

\section{Image quality and subject movement}

Metallic objects, even non-ferromagnetic ones, are known to cause image artifacts due to magnetic susceptibility. These 'susceptibility artifacts' are signal voids in and around the objects and they reduce the signal to noise ratio. Susceptibility artifacts are produced from the alteration of magnetic fields in the presence of magnetically susceptible objects. Given this, it is necessary to consider potential image artifacts when introducing EEG electrodes and cables into the MR environment. Since magnetic susceptibility is a function of the applied magnetic field, susceptibility artifacts increase with stronger MR field strengths.

These issues have currently been addressed by using materials with less magnetic susceptibility and by improving electromagnetic shielding. ${ }^{21}$ Many centers currently use commercially available elastic cloth caps with built-in plastic or carbon electrodes attached to carbon fiber leads. The electrodes and initial length of the leads are then incorporated into the 
fabric, which simplifies patient set-up (Figure 2). Newer developments include the use of conductive silver-carbon ink microstrips, which show hope for further improvement of signal to noise ratio at high magnetic field strengths. ${ }^{35}$

Another major cause of image degradation is patient motion during scanning. Head movements as little as $5 \mathrm{~mm}$ can produce significant artifacts that cannot necessarily be corrected by motion correction paradigms during post processing of images. As EEG-fMRI studies of epilepsy may require longer scan times (up to one to two hours), subject comfort is an important factor to consider in order to limit movement. Patient discomfort can arise due to pressure from the elasticity of an EEG cap or from the weight of the head being placed on relatively few electrodes in the occipital region of the head. In order to address the pressure issue, our center has modified our EEG cap to include strips of dense padding in the occipital regions to distribute the weight of the head over a larger surface area. Many centers also use adjustable foam cushions placed around the head or vacuum head cushions filled with polystyrene beads to minimize head movement and improve comfort. ${ }^{36}$

\section{EEG-fMRI PROTOCOL AND EEG ARTIFACT REMOVAL Gradient artifact}

Acquiring EEG during MR imaging results in high amplitude artifact in the EEG recording due to rapidly switching gradients, called the gradient switching artifact, or gradient artifact. Figure 3 shows an EEG trace recorded during scanning that is unreadable prior to gradient artifact removal. To avoid these artifacts, early EEG-fMRI studies were performed using a 'spike-triggered' approach where one brain volume of MR images (typically 20-24 slices) are acquired immediately after an epileptiform discharge is seen during online viewing of EEG acquired in the static MR field [Figures 1 and 4 , see also ${ }^{9-11}$ ]. A similar number of images are also acquired during baseline periods, during which no interictal discharges are seen. Spiketriggered fMRI prevents the need for gradient artifact removal from the EEG signal, yet still captures the hemodynamic response peak that occurs several seconds after each spike (Figures 1 and 4). Note that it typically takes $1.5-3 \mathrm{sec}$ to acquire one fMRI image of the entire brain, during which time one measurement of BOLD signal is made for every voxel in the brain. Although used with considerable success, spike-triggered fMRI prevents the detection of possible discharges during imaging, decreases the sensitivity of spike detection, and image acquisition at irregular time intervals requires a long refractory time for complete magnetic field relaxation. ${ }^{21}$

Refinement of recording systems and EEG processing techniques by several research groups has since allowed EEG and fMRI to be performed continuously and simultaneously in humans and animals. ${ }^{21,37-40}$ Figure 4 shows a comparison between the spike-triggered and continuous MR image acquisition techniques. Most artifact removal techniques are performed by calculating an average of the gradient artifact waveform in each EEG channel and then subtracting it from the recorded EEG. ${ }^{21,37,38}$ It is important to note that to do this, the EEG sampling frequency rate is increased from standard clinical rates $(\sim 200 \mathrm{~Hz})$ to more than $1000 \mathrm{~Hz}$. In this way, it is possible to get a detailed definition of stereotypical, repetitive MR

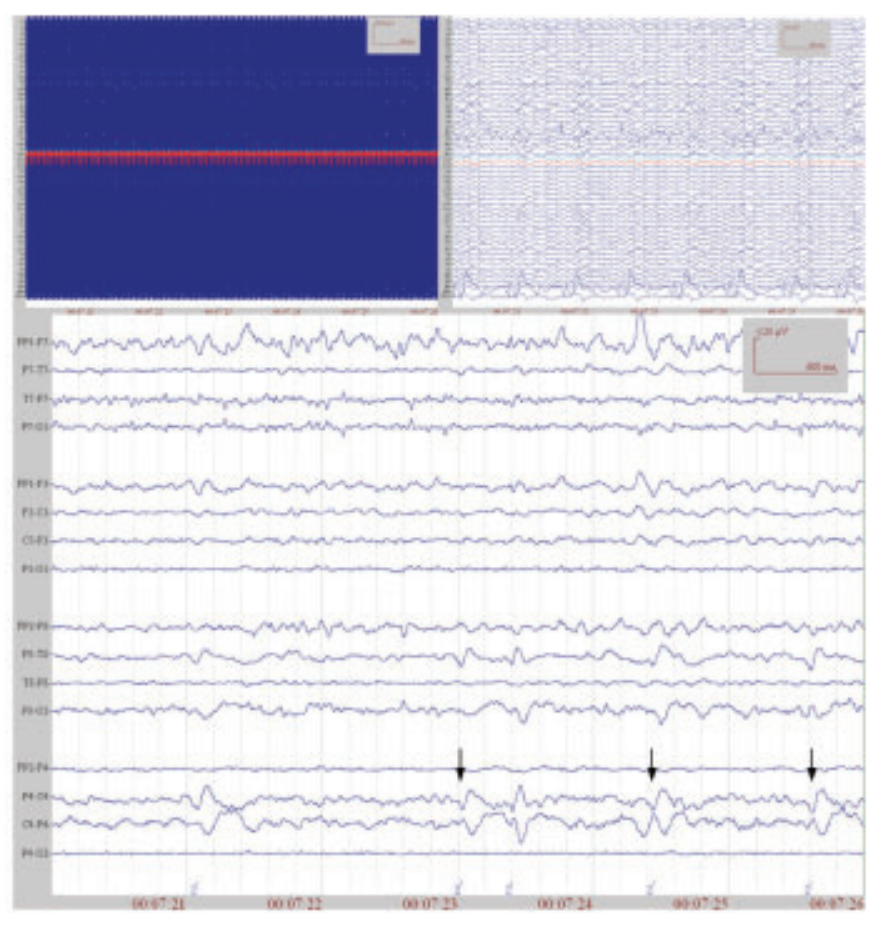

Figure 3: EEG recordings during MR scanning. Top Left. Unreadable EEG recording during scanning. Top Right. The same EEG segment with $M R$ artifact removed. Bottom. The same EEG segment after MR and ballistocardiogram (BCG) artifact removal and application of a bipolar montage. Several interictal discharges are identified by arrows.

gradient artifacts in EEG recordings, which can then be removed using post-processing techniques. . $^{21,37,38}$

\section{Pulse artifact}

Artifact in EEG resulting from the cardiac cycle is called ballistocardiographic artifact (BCG), or pulse artifact, and is highly variable between patients. These artifacts probably result from subtle movement of the scalp and EEG electrodes associated with rapid blood flow through scalp arteries. BCG removal techniques commonly involve the determination of an average BCG-associated waveform (based on the study at hand) and the subtraction of it from each channel ${ }^{41}$ This is theoretically similar to the gradient artifact removal techniques described earlier. Another similar technique involves applying a mathematical representation of the artifact waveform as a spatial filter to the EEG data. ${ }^{42}$ In order to remove BCG artifact using these techniques, the timing of heartbeats must be determined using a pulse oximeter or a two-lead electrocardiogram. Independent component analysis and principal component analysis have also been applied to identify the components related to $\mathrm{BCG}$ and removing them from the EEG. ${ }^{43-45}$ While independent component analysis may improve the reduction of BCG artifact over an average subtraction method, ${ }^{43}$ both approaches have been used with considerable success. 


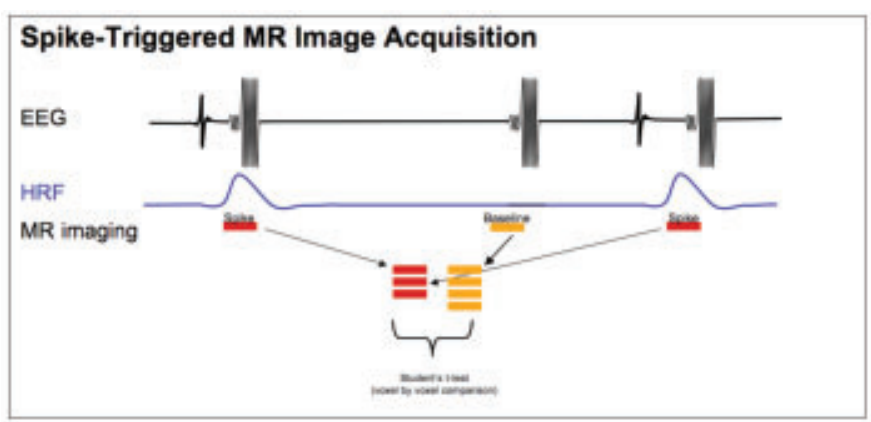

Continuous MR Image Acquisition

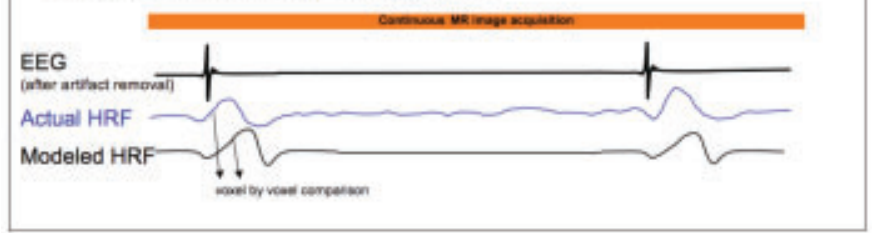

Figure 4: Summary of 'spike-triggered' versus 'continuous' study designs for EEG-fMRI experiments. Spike-triggered designs compare $M R$ signal changes measured directly following an epileptiform discharge to a baseline period when no discharges are present. Continuous designs compare the actual MR signal acquired continuously throughout a study to a modeled HRF on a voxel by voxel basis.

There has been a recent focus on more accurate characterization of the BCG in order to improve its removal. BCG and motion artifact can be well characterized by measuring the voltage induced in a number of wire loops attached to the EEG cap during scanning, allowing for effective removal of the artifact without degrading the EEG signal. ${ }^{46}$ Another approach proven to be effective in improving artifact removal is the synchronization of the internal timing clocks of the EEG and MRI systems. This allows a better characterization of the artifact waveform at a lower sampling rate and may increase the useable EEG bandwidth to $0-150 \mathrm{~Hz}$, instead of the typical $0-50 \mathrm{~Hz}{ }^{47}$

Currently, many centers perform gradient switching artifact and $\mathrm{BCG}$ artifact removal on-line, allowing real-time visualization of the EEG during MR image acquisition. Figure 3 provides an example of unreadable EEG collected during scanning, the same recording after gradient artifact removal, and after BCG artifact reduction.

\section{fMRI collection and data analysis}

Functional MR images are typically acquired simultaneously with scalp-EEG in epilepsy patients for around 45-120 minutes. ${ }^{48}$ The benefit of longer scan times is the potential of capturing more interictal discharges. We routinely sleep-deprive patients and occasionally withhold morning anticonvulsant medications (provided the risk of a prolonged seizure is not too great) in an attempt to capture more interictal discharges.

Following fMR image acquisition, preprocessing of the image data is carried out. Motion correction techniques are employed to realign all images within a series of MR images to the first one; however, this may not be sufficient, and using motion correction as regressors in statistical modeling may be necessary. ${ }^{49-51}$ Spatial smoothing in the form of a Gaussian filter is also typically performed to reduce noise. In addition, low frequency drifts in MR data caused by scanner inconsistencies ${ }^{52}$ need to be corrected using a third order polynomial, although this may not always be sufficient. ${ }^{53}$ Other approaches to fMRI baseline signal correction include principal component analysis ${ }^{54}$ and quantifying magnetic field drift by detection of resonance frequency before and after fMRI acquisition. ${ }^{55}$ Prior to analysis of the fMRI data, interictal discharges are identified by a certified electroencephalographer and are used as 'events' for subsequent event-related fMRI analysis. Many studies utilize more than one electroencephalographer and/or blind those reading the experimental EEG from patient information in order to decrease potential biases in identifying interictal discharges. Following discharge identification, the timing and duration of these events are then transformed into the time frame of the fMRI dataset as expressions of scan repetition time (TR). An expected hemodynamic response for the entire dataset is then generated from the convolution of the binary timing/duration of the events and the theoretical HRF to a single impulse of neuronal activity. The measured time-varying signal from each voxel in the brain is statistically compared to this modeled HRF. A greater correlation with the modeled hemodynamic responses indicates a greater likelihood that a particular voxel is activated in association with the event of interest (interictal discharge). The degree of statistical correlation is then displayed using a color-coding scheme that is overlaid onto a higher resolution anatomical brain image, often called statistical parametric mapping.

In spike-triggered experiments, MR scanning only occurs for a few seconds following an identified interictal discharge (Figure 4). As a result, only a portion of the actual HRF is measured. Analysis of data from spike-triggered EEG-fMRI experiments involves a Student's $t$ test of the difference in mean intensity of a voxel between images collected after the spike and during baseline (Figure 4). This $t$-statistic map is then overlaid onto the higher resolution anatomical images. Positive t-statistics (group of spikes greater than baseline) and negative $t$-statistics (baseline greater than groups of spikes) are calculated to show areas of spike-associated BOLD increases and areas of reduced BOLD signal, respectively. Similarly, continuous EEG-fMRI experiments use positive and negative t or F-statistics to generate maps based on how similar the BOLD signal in a particular voxel is to a model of spike-related signal change. In this way, the statistical strength represents the likelihood that a particular voxel is activated (or deactivated) in relation to an interictal discharge.

Data-driven analyses of simultaneous EEG-fMRI data have also been developed to decrease reliance on purely hypothesisdriven approaches. ${ }^{56}$ One novel analysis method combines temporal independent component analysis of EEG data to identify discharges, with spatial independent component analysis of imaging data to localize BOLD responses in order to investigate the relationship between interictal discharges and BOLD signal in an animal model of epilepsy. ${ }^{56}$ Using this method, it has been found that a linear transformation model fairly accurately represents the relationship between interictal discharges and BOLD fMRI signal. ${ }^{56}$ 


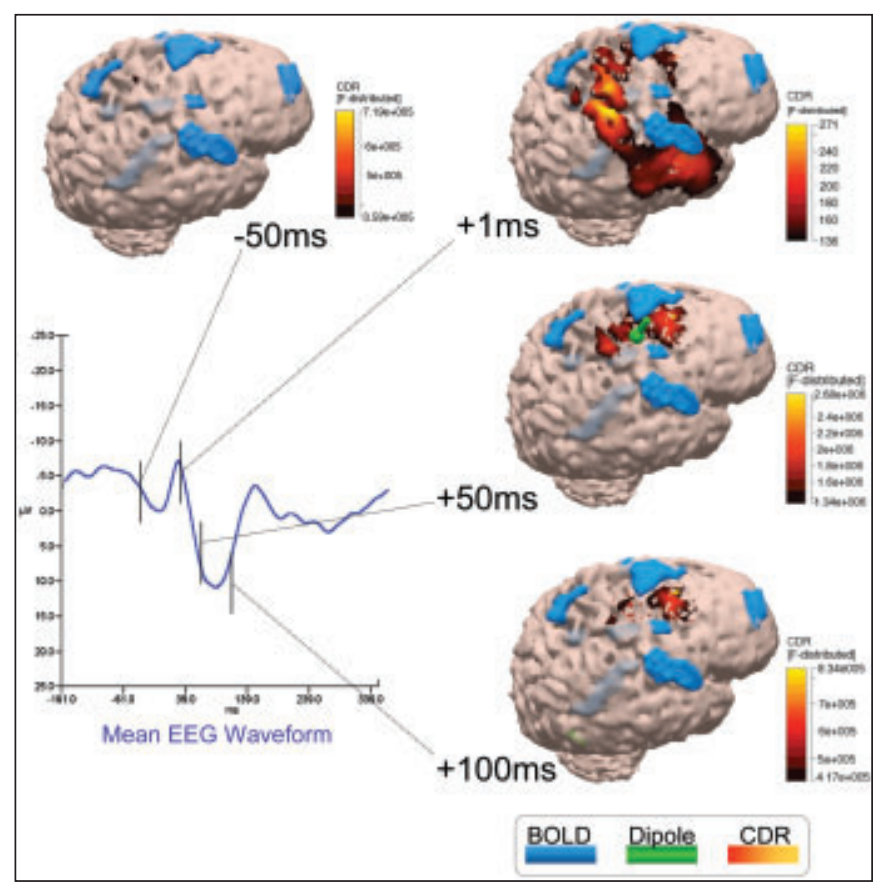

Figure 5: Co-localization of dipole source, current density reconstruction, and BOLD signal overlaid onto a $3 D$-model of a single subject's brain from a 60 minute EEG-fMRI recording. Line plot shows the average EEG waveform calculated from all interictal discharges recorded. Surrounding figures show the mean BOLD activation $(t>3.1)$ for all interictal discharges (blue). The orange/red surface color maps show the current density reconstruction $(C D R)$, and the green ball and stick figure display the moving dipole at different time points of the mean EEG discharge. The brain image data displayed are from time $=-50 \mathrm{~ms}$, $+1 \mathrm{~ms},+50 \mathrm{~ms}$, and $+100 \mathrm{~ms}$ relative to the point of maximum negativity of the mean EEG waveform.

\section{Source Localization}

Data-driven techniques for fMRI analysis continue to develop in concert with conventional EEG-driven approaches. Thus far, most studies use EEG data primarily to identify the time points of interictal discharges to use in subsequent event-related fMRI analysis. However, EEG data can be used further to provide information about the area producing interictal discharges by source localization techniques. ${ }^{57}$ Many EEG localization techniques use a boundary element method, which attempts to model the electrical conductance and impedance of the human head based on the properties of various tissue layers. A fine cubic grid is then projected onto an image of the brain, creating points representing potential electrical sources at each intersection of the three-dimensional grids. ${ }^{58}$ Dipole analyses calculate possible sources of electrical activity in the brain as it changes over time (moving dipole), or averaged over a certain time period (stationary dipole). From a number of discrete calculated dipoles, a current density reconstruction can be created which maps the dipole moment per unit volume and is overlaid onto the surface of a model of the cerebral cortex. Figure 5 shows an EEG current density reconstruction and dipole localization at various time points of the average of all interictal spike waveforms recorded in one hour of EEG-fMRI in a subject with a right posterior temporal periventricular nodular heterotopia. The mean BOLD response to all interictal discharges recorded is overlaid onto the same brain for comparison. These data show concordance between the different modalities that is good, but not complete, and which may colocalize more at some time points in the EEG discharge than others.

\section{EXPERIMENTAL RESULTS}

By combining fMRI and EEG, research groups have focused on improving non-invasive methods of localizing the epileptogenic zone in patients with a variety of focal and generalized epilepsies. The EEG and fMRI measure different aspects of cerebral activity, and several groups have attempted to determine the extent to which the signals co-localize.

The initial aims of early spike-triggered EEG-fMRI studies were to establish whether EEG could be recorded in the MR scanner and if interictal discharges measured by EEG elicited a measurable BOLD response. ${ }^{9,20}$ It should be noted that the MR image acquisition, EEG filtering techniques and hardware (EEG apparatus and MRI scanners) might differ both between research centres and in the same centre over time. This will be noted where relevant. The following sections will summarize results from a variety of, but not all, EEG-fMRI studies.

\section{Focal discharges}

\section{Early findings from EEG-fMRI experiments}

The first attempt to study the localization of interictal discharges using spike-triggered fMRI involved multiple investigations of ten patients. ${ }^{11}$ The authors recorded between 21 and 50 interictal discharges per experiment. Reproducible focal BOLD signal changes that were largely concordant with EEG spike localization were seen in six patients. Three patients showed no significant BOLD activation, and one patient failed to show reproducible activation in a subsequent investigation. Another spike-triggered fMRI study published the same year demonstrated spike-associated BOLD signal changes that were correlated with EEG discharge localization in nine of ten subjects. ${ }^{59}$ A study of eleven patients examined the spatial correlation between spike-associated BOLD signals and a variety of presurgical investigations including scalp and intracranial video EEG monitoring, structural MRI, positron emission tomography, and single photon emission computerized tomography, and neuropsychological testing. ${ }^{24}$ Overall, the BOLD response showed good spatial concordance with clinical findings in eight of eleven patients in total, and in five of the six patients who had subsequent intracranial EEG recordings. Using spike-triggered fMRI, BOLD signal changes have been found in areas that are consistent with clinical presentation of specific epilepsy syndromes. ${ }^{60,61}$

The first report of continuous EEG of diagnostic quality during fMRI was in a group of ten patients with focal epilepsy. ${ }^{62}$ After exclusion of five patients due to lack of discharges, superfluous discharge rate (resulting in inadequate baseline), and motion, the remaining five subjects all showed BOLD signal increases that were temporally correlated to spikes detected with EEG. In this study, the authors found peak signal intensity sixseven seconds after spike detection. ${ }^{62}$ 
A larger report of 48 fMRI studies performed on 38 patients with partial epilepsy using either spike-triggered or continuous EEG-fMRI showed significant BOLD activations in 22\% (2/9) and $45.5 \%(10 / 22)$ of the experiments, respectively, after removal of studies with no EEG discharges or with complicating issues such as subject movement. ${ }^{36}$ The yield over all studies was $38.7 \%$ (12/31studies). ${ }^{36}$ Based on these and other results, continuous EEG-fMRI has been deemed more sensitive to the detection of discharges than spike-triggered fMRI. This study also showed that BOLD activation was more likely for subjects with bursts of discharges, rather than isolated discharges. In the 12 patients with BOLD signal changes, a total of 38 different regions showed increases in signal. The 29 sites of largest activation volume were concordant with EEG localization in all but one case. Subsequent intracranial EEG recordings in four patients further validated the EEG-fMRI results. ${ }^{36}$

Animal models of focal epilepsy have also been developed to allow intracranial EEG monitoring with simultaneous fMRI. ${ }^{63}$ These techniques can be used further to investigate spatial concordance between modalities and explore the physiological basis of each signal.

\section{Negative BOLD changes}

The EEG-fMRI studies first examined BOLD signal increases (activations) only. Negative BOLD responses, sometimes called deactivations, are now routinely examined in patients with focal and generalized epilepsy. ${ }^{64-67}$ A large review of EEG-fMRI studies of focal epilepsy reported deactivations in 26 of 34 experiments that demonstrated robust BOLD responses to focal discharges, and noted deactivations were often found with concomitant activations. ${ }^{48}$ There was spatial concordance of deactivations and EEG localization in 16 patients, which is less than the typical concordance between activations and EEG localization. It was noted that isolated spikes were more likely to produce activations or deactivations individually, whereas bursts of spikes were more likely to produce both activations and deactivations together.

There are several possible mechanisms underlying the negative BOLD response. One potential mechanism is that neurovascular coupling in regions showing deactivation is altered, resulting in an increase in cortical oxygen metabolism, without a concomitant increase in cerebral blood flow, thus producing an apparent decrease in BOLD signal. This explanation is similar to a common interpretation of the "initial dip' measured in regions exhibiting a positive BOLD response. Another potential explanation is that cerebral blood flow is decreased in areas showing decreased BOLD signal due to a vascular steal phenomenon, where blood flow is diverted from areas showing deactivation to areas of activation. ${ }^{68}$ Deactivation may also be due to suppressed neuronal activity at a given site, which in turn, is associated with reduced oxygen metabolism and blood flow. Overall, a number of studies have inconclusively investigated this issue $\left[{ }^{48,68-70}\right.$ for details], suggesting that multiple mechanisms underlie BOLD deactivations whose interactions may be complex.

\section{Cortical and subcortical networks}

Another phenomenon repeatedly observed is that BOLD signal changes can occur at sites distant to the presumed seizure focus in focal epilepsies. Several reports have focused on characterizing the involvement of distant cortical and subcortical structures in focal interictal discharges. An EEG-fMRI study of six patients with malformations of cortical development found BOLD changes in all patients, with five patients displaying BOLD signal changes at sites distant to the presumed epileptogenic region ${ }^{66}$ (Figure 6). Three patients also showed BOLD signal changes in subcortical structures such as the

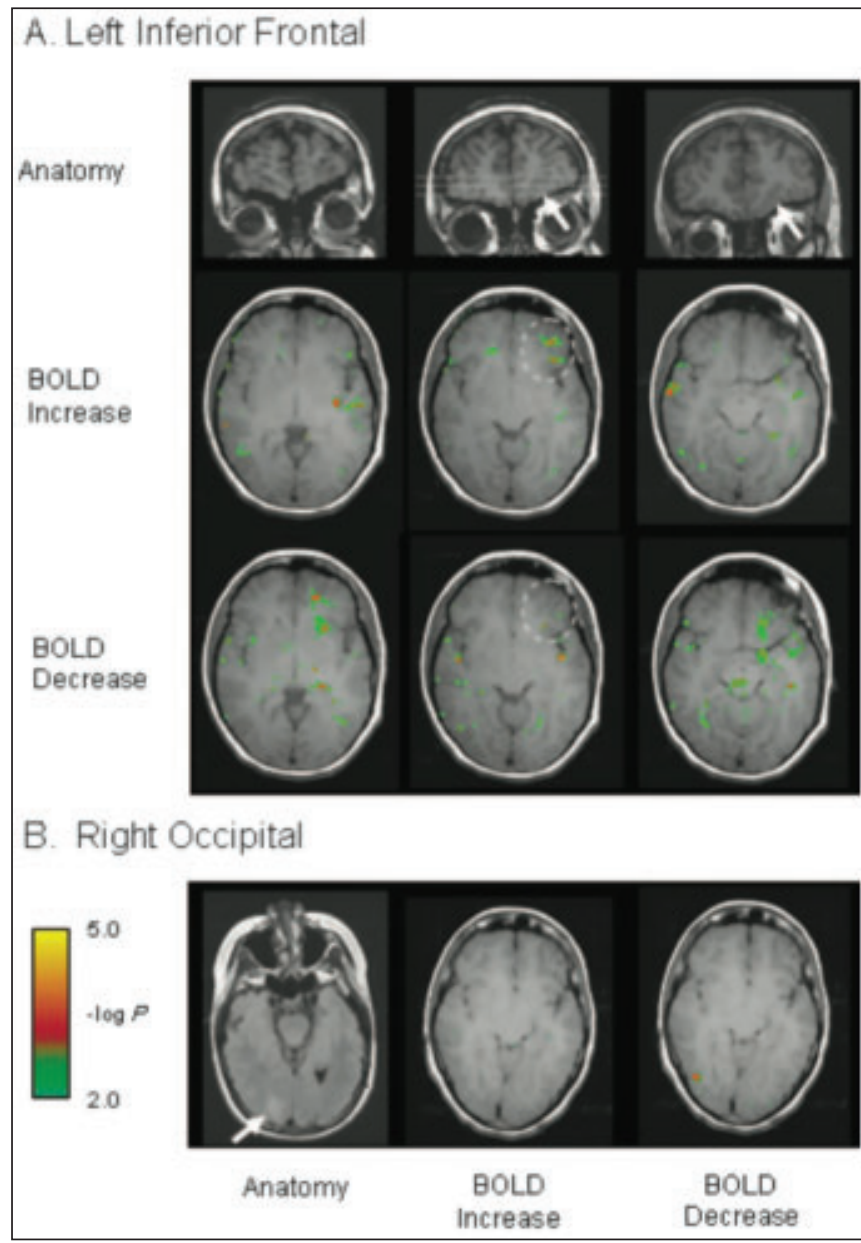

Figure 6: Lesional and perilesional blood oxygen level-dependent (BOLD) signal changes associated with interictal discharges in two subjects. A. Patient with refractory frontal lobe epilepsy. Top row: coronal T1-weighted images showing site of cortical dysplasia (arrows). Middle row: maps of BOLD signal increases overlaid onto the corresponding $T 1$ axial image (5mm between slices). BOLD signal increases were seen within the lesion and in the ipsilateral insular cortex. Bottom row: maps of BOLD signal decreases overlaid onto the corresponding $T 1$ axial image (5 $\mathrm{mm}$ between slices). BOLD signal decreases were seen peri-lesionally or at the margin in slices immediately above and below. The dashed circles in the axial images indicate the approximate site of the lesion. B. Patient with refractory occipital lobe epilepsy. The arrow in the left image shows the lesion seen on fluid-attenuated inversion recovery images. The only cortical BOLD signal change was a perilesional BOLD signal decrease (right image). Modified from Federico et al. Neurology 2005;64(7):1125-1130. 
brainstem reticular formation, basal ganglia or thalamus. These findings are consistent with some animal studies that show subcortical involvement in cortical epileptiform discharges. ${ }^{71-73}$ In a feline model of epilepsy, epileptiform discharges have been detected in subcortical structures prior to cortical detection of the discharges. ${ }^{74}$

A report of 35 EEG-fMRI studies in 27 patients with temporal lobe epilepsy showed BOLD signal increases at sites distant to the seizure focus in most patients. ${ }^{75}$ Specifically, $83 \%$ of studies had BOLD responses that predominated in the spiking temporal lobe (activation or deactivation); however, BOLD responses were often seen in contralateral homologous temporal lobe and extratemporally. Another study of two patients with reading epilepsy using spike-triggered fMRI found fMRI activation of bilateral cortical and subcortical areas associated with readinginduced epileptiform discharges in one subject, but no fMRI activation in the second, likely due to the fact that only four spikes were recorded in this patient. ${ }^{61}$

Based on these studies and others, it is likely that distant cortical and subcortical circuits are involved in the generation of 'focal' discharges. In fact, it may be the case that electrical changes may occur in more than one brain region, which may not be appreciable with EEG alone as demonstrated in a study of benign epilepsy with centro-temporal spikes. ${ }^{60}$ The precise role of the structures in ictogenesis is unclear and should be tested further by surgical trials assessing the effect of resecting cortical areas showing BOLD signal on post-surgical outcome.

\section{Hemodynamic response}

Most publications, particularly those described earlier, were based on the presumption that epileptiform discharges are associated with a standard and predictable hemodynamic response. This may not be true. Analyzing EEG-fMRI data using a HRF specific to the phenomenon or patient rather than to a standard (canonical) hemodynamic response may increase the detection rate in an EEG-fMRI study. It has been suggested that fMRI data should be analyzed using several different HRF with peaks between three to nine seconds to capture activations with different peak times and to capture deactivations that might occur at slightly different times compared to activations. ${ }^{76}$

To this end, EEG-fMRI data from 31 patients were analyzed using multiple HRF peak times at three, five, seven, and nine seconds, in addition to a 'standard' 5.4-second peak. ${ }^{64} \mathrm{By}$ analyzing results using multiple HRF peak times, the detection of BOLD signal activation improved from $45 \%$ to $62.5 \%$ of datasets. It was also shown that negative BOLD responses typically occurred with a longer time to peak than positive BOLD responses, typically nine seconds. ${ }^{64}$

Further improvements in the sensitivity of detecting activation changes have been reported by measuring variable HRFs from individual epileptic spikes, ${ }^{53}$ and by using patientspecific HRFs ${ }^{77}$ or voxel-specific HRFs in data analysis. ${ }^{78}$ These studies have attempted to improve modeling of the HRF because it may differ slightly between subjects and between brain regions in the same subject.

Recently, it has been suggested that BOLD changes can occur prior to the detection of interictal spikes detected on EEG. ${ }^{79}$ Sixteen EEG-fMRI studies were analyzed using seven HRF peak times of three or one second prior to the discharge as well as one, three, five, seven, or nine seconds after the discharge. Interestingly, analysis of five of the EEG-fMRI sessions showed spikes with a maximal HRF peak that occurred around one second after the EEG spike. This suggests that in these patients, the physiological processes underlying spike-associated BOLD signal changes may begin before electrical activity can be detected using scalp EEG. ${ }^{79}$ This has also been demonstrated in an animal model. ${ }^{80}$

\section{BOLD signal concordance with EEG}

Blood Oxygenation Level Dependent activation changes are generally concordant with localization of epileptiform discharges using EEG and other modalities. ${ }^{9,10,23,36,53,64,66,81,82} \mathrm{~A}$ recent report of 63 patients with focal epilepsy compared the concordance of spike-associated BOLD changes with the best available electroclinical data from ictal EEG, interictal EEG, and structural MRI. ${ }^{40}$ Of the 63 patients studied, 34 had discharges measured by EEG, 23 of which had significant BOLD change associated with the discharges (ten of these had positive BOLD changes only, four had negative BOLD changes only, and nine had both positive and negative BOLD changes). The BOLD activations were concordant with the presumed seizure focus in 15 of the 17 patients that had clear clinical seizure localization and a measurable BOLD increase. The BOLD deactivations were concordant with presumed seizure focus in only three of ten patients with measurable BOLD decreases. ${ }^{40}$ An additional two patients with negative BOLD changes had uncertain clinical localization. Other studies have also shown that deactivations are less concordant with spike localization, ${ }^{75}$ supporting a distinct electrophysiological mechanism from activations ${ }^{40}$ (see earlier).

Although localization of epileptic regions by fMRI has been previously confirmed with intracranial recordings, ${ }^{24,36}$ a recent study was the first to systematically quantify the concordance between the two modalities. ${ }^{58}$ Scalp EEG-fMRI and intracranial EEG (done on a separate occasion) was performed on five patients. By locating scalp EEG peaks using source localization and fMRI peaks using conventional analysis, it was observed that both modalities were more concordant with intracranial EEG individually than when compared to each other. For instance, intracranial electrodes typically showed at least one active contact during an interictal discharge if they were within 20-40 $\mathrm{mm}$ of the location of a scalp EEG or fMRI peak. The fact that each technique provides complementary information about interictal discharge localization supports the continued combination of EEG and fMRI to investigate epileptic phenomenon. Another interesting observation was that an increase in low frequency activity in the intracranial recordings $(0.5-4 \mathrm{~Hz})$ was seen in contacts at sites corresponding to BOLD signal increases, suggesting that slow waves may influence the energy requirements of neurons, but further investigation is needed. . $^{58}$

There is some controversy about the extent to which scalp EEG and fMRI are expected to colocalize, since each modality is sensitive to different physiological factors and since they have significantly different spatial and temporal resolutions. Scalp EEG measures the summed, synchronous activity of cortical pyramidal cells and does this with a millisecond temporal resolution. Its unit of measure is voltage. Scalp EEG is particularly sensitive to dipole moment vectors produced at right 
angles to the scalp close to the cortical surface (i.e., from neocortical pyramidal cells) and the amplitude of a discharge on EEG is primarily influenced by the orientation/geometry of the current source, the area of the source, as well as the synchrony of elements within the volume. ${ }^{83}$ An area of cerebral cortex greater than $10 \mathrm{~cm}^{2}$ is required to be activated to produce a measurable scalp EEG change ${ }^{84,85}$ As a result, epileptiform activity may not appear on scalp EEG until it has involved sufficient tissue area or developed enough synchrony. It has also been shown that scalp EEG recordings can represent sites of seizure propagation instead of seizure initiation (as measured with intracranial EEG) in some patients, particularly with mesial temporal lobe seizures, or fail to detect ictal activity completely in others. ${ }^{86}$ Due to this fact, only the largest interictal discharges are apparent on scalp EEG, ${ }^{84}$ and scalp EEG may therefore not be entirely representative of the epileptogenic zone (defined as the minimum tissue volume whose removal is necessary to ablate seizure..$^{87}$

Thus, the complex relationship of scalp EEG to intracranial source generators precludes fMRI from completely colocalizing with it, but there still remains significant overlap. Functional MRI is sensitive to metabolic changes in a given brain volume, so BOLD responses to epileptiform discharges may involve areas of discharge generation and/or propagation. In addition, fMRI provides a measure of brain activation throughout the entire brain (unlike scalp EEG) with a greater spatial resolution that is limited by voxel size (approximately $5 \mathrm{~mm}^{3}$ ). Furthermore, fMRI is sensitive to non-neuronal as well as neuronal activity changes. For example, high levels of asynchronous synaptic activity in glial cells may cause focal hemodynamic changes that are evident with fMRI, without changing the EEG. ${ }^{83}$ Therefore, it is not surprising that scalp EEG and fMRI may provide discordant results at times since fMRI can identify regions of spike-associated neuronal or glial activation that may be too small, too asynchronous, or too deep to be detected by scalp EEG.

Differences in reported concordance between EEG and fMRI data can also result from various technical issues. For example, differences in scanner field strengths between studies affects fMRI signal to noise ratio, and thus can result in differences in the quantity and location of BOLD responses that are detected. Differences between individual subjects (e.g., variable number of spikes included in a study) will result in differences in the statistical power. Specifically, patients with fewer spikes may not have as many areas showing BOLD signal changes or changes with lower magnitudes due to fewer spikes to include in the statistical analysis of spike-associated BOLD signal change..$^{23,36,75}$ Furthermore, each patient or brain region may have a different hemodynamic response. This should be identified and modeled in order to increase the statistical power of the study and increase the detection of spike-associated BOLD signal changes. ${ }^{78,88}$ Other issues include the fact that, despite the many technical advances in the EEG-fMRI field, the quality of EEG recorded during scanning in most centers is good, but not identical to that recorded outside the scanner. As a result, smaller epileptiform discharges may be missed, thereby reducing the statistical power of the study. It has also been reported that different types of epileptiform discharges may have different BOLD responses ${ }^{65}$ and they should be grouped separately, although most studies to date have not done so.

\section{EEG-fMRI in the clinical setting}

Ictal scalp EEG data are used in the clinical setting to make surgical decisions as interictal data may localize to the ictal onset zone, but also to other brain regions. ${ }^{87}$ Indeed, interictal discharges can be seen in irritative zones in the brain that may not necessarily be critical in the genesis of seizures. It has been shown that in the majority (but not all patients) with focal epilepsy, interictal discharges outside the presumed seizure focus can decrease in frequency after resection of the ictal onset zone. ${ }^{87}$ Thus, it can be seen that while interictal discharges are a marker of epileptogenicity, they do not necessarily localize to the ictal onset zone. In a subset of patients, scalp EEG may not produce localizing ictal recordings (e.g., no clear onset seen, seemingly multifocal onset zones, etc), particularly in those with deep seizure foci, such as in mesial temporal or frontal lobe epilepsy. Consequently, intracranial EEG recordings of seizures become clinically indicated for further confirmation of the ictal onset zone. Compared to intracranial EEG, both scalp EEG and functional MRI remain less powerful techniques to definitively identify the seizure onset zone. Studies comparing spatial localization of the irritative zone using scalp EEG and fMRI to intracranial EEG and to each other have indicated that scalp EEG and fMRI both colocalize more closely with intracranial localization alone then they do with each other. ${ }^{58}$ This suggests that scalp EEG and fMRI provide complementary information regarding the intracranial source of an epileptiform discharge; however further work validating scalp EEG-fMRI against intracranial EEG recordings is necessary to improve clinical application of the technique. For instance, no studies to date have systematically studied the relationship between the pre-surgical BOLD activation and the surgical resection site and postoperative outcome.

The clinical role of EEG-fMRI is expanding. Recently, 29 patients were studied who were previously rejected for epilepsy surgery because of an unclear seizure focus or presumed multifocality. ${ }^{89}$ These patients underwent EEG-fMRI studies and eight patients were re-evaluated for surgery because of the results of the studies. They underwent surgery with variable outcomes. In addition, EEG-fMRI was used to confirm that some patients were not suitable for surgery due to multi-focal pathology or extensive subcortical involvement. The authors suggest EEG-fMRI should play a role in seizure focus localization when results from other investigations are inconclusive, as well as to confirm multifocal epilepsy in order to prevent surgeries that may have minimal chance of success. ${ }^{89}$

\section{Focal discharges: summary}

Blood Oxygenation Level Dependent signal changes have been found in $40-80 \%$ of patients studied with focal epilepsy, and concordance of the BOLD signal location with EEG localization varies from 50 to nearly $100 \% .^{36,40,90,91}$ The differences in concordance can be attributed to statistical modeling and methodological differences between studies, as well as differences in BOLD and EEG localizations. ${ }^{56}$ Improvements in analysis techniques and a more complete understanding of the relationship between epileptiform discharges and BOLD signal, may allow detection of BOLD changes with up to $100 \%$ of spikes. ${ }^{56}$ Most centers now routinely 
perform continuous, simultaneous EEG-fMRI, which has a greater sensitivity than spike-triggered protocols. Much of the work up to this point has been performed at 1.5 Tesla, using 32 or less channels of EEG. Further investigations with higher MR field strengths ( $\geq 3$ Tesla) should allow the detection of BOLD signals associated with smaller amplitude discharges. In addition, using more EEG electrodes in EEG-fMRI simultaneous recordings would improve EEG source localization. By investigating sub-populations of patients with more homogenous forms of epilepsy, it is hoped that various epilepsy syndromes can be more closely characterized. Indeed, a number of recent studies have investigated children with specific epilepsy syndromes in order to prove feasibility and applicability of EEGfMRI in this population. ${ }^{92-94}$

\section{Generalized discharges}

The first EEG-fMRI study of patients with generalized spikeand-wave (GSW) discharges came from Hill and colleagues, ${ }^{95}$ who studied patients with generalized discharges induced by photic stimulation. They observed BOLD signal increases in the occipital lobe of patients compared to controls during photic stimulation, regardless of whether epileptiform discharges were present or not. BOLD signal changes were not observed elsewhere, most notably the thalamus.

Archer et al, ${ }^{67}$ first showed consistent areas of deactivation and activation associated with GSW discharges in a group of five patients using spike-triggered EEG-fMRI at 3 Tesla. Deactivation was seen in four patients and it was consistently located in the posterior cingulate gyrus. Variable BOLD activation was detected in the same four patients in the precentral sulci bilaterally. ${ }^{67}$

Continuous fMRI collection with simultaneous EEG was first applied to generalized epilepsy in a single patient with frequent absence seizures associated with $3-\mathrm{Hz}$ GSW. ${ }^{96}$ The thalamus exhibited focal activation during the runs of $3-\mathrm{Hz}$ generalized discharges recorded in addition to widespread cortical deactivation that was maximal frontally. ${ }^{96} \mathrm{~A}$ more recent report of continuous EEG-fMRI datasets collected from a group of 15 patients with GSW was performed to better characterize spikeassociated thalamic and cortical BOLD signal changes. ${ }^{76}$ The BOLD signal changes were seen in 14 patients (90\%), with bilateral thalamic changes seen in 12 patients (80\%), and activation predominating over deactivation in the thalamus. In subjects with measurable cortical BOLD changes, deactivation predominated in nine patients and activation in five patients. ${ }^{76}$ The cortical BOLD changes were seen to the same extent both in the frontal and posterior head regions, which was not consistent with the typical anterior to posterior predominance of the GSW discharges seen in these patients using EEG.

A group analysis was later performed using the same patient datasets. ${ }^{97}$ From this analysis, activations were seen bilaterally in the thalamus (which is traditionally believed to be critical for generating GSW discharges) as well as bilaterally insulae, mesial midfrontal regions, and cerebellum. ${ }^{97}$ Deactivations were seen bilaterally in the posterior cingulate gyrus, as demonstrated previously, ${ }^{67}$ as well as in anterior frontal and parietal lobes. The location of the frontal and parietal deactivations in this study corresponded closely to sites of activation seen in the 'default state'. ${ }^{97,98}$ The default or resting state refers to a pattern of activation associated with a wakeful resting state, which is suspended temporarily once a task is presented to a subject. ${ }^{98}$ It is believed that thalamic activation (and therefore frontal and parietal BOLD deactivation) associated with GSW discharges may cause the momentary interruption of the resting state of the brain. This, in turn, may explain transient reductions of responsiveness seen in some patients during GSW discharges. ${ }^{67,97}$ Other recent studies have found similar deactivations of resting state networks in combination with activations of discharge producing regions. ${ }^{48,99}$ It was also found in a study of four subjects with generalized epilepsy that cerebral blood flow (measured with MR arterial spin labeling) and BOLD signal increase linearly suggesting neurovascular coupling is preserved during these epileptic events. ${ }^{99}$ Further work has demonstrated decreases in resting state activity as a consequence of interictal discharges in temporal lobe epilepsy subjects who have complex partial seizures. ${ }^{100}$ Subjects with extra-temporal lobe epilepsy were not found to have the same suspension in resting state.

Many animal studies have investigated BOLD changes in response to generalized discharges/seizures induced by drugs, ${ }^{101-}$ 104 genetic manipulations, ${ }^{105}$ or both. ${ }^{106}$ Overall, these studies have corroborated evidence from electrophysiological recordings and human EEG-fMRI studies that show extensive thalamic activation leading up to and during absence seizures. ${ }^{101,102,104,106}$ However, the patterns of positive and negative BOLD changes in the neocortex in animal studies are not entirely concordant with human data. Positive BOLD changes are more localized and have been seen in the frontoparietal cortex ${ }^{106}$ sensorimotor cortex ${ }^{101}$, sensory and parietal cortices (with mixed negative BOLD responses), ${ }^{102}$ and retrosplenial cortex ${ }^{104}$ in various animal models of GSW discharges/absence seizures, compared to the mesial midfrontal and insulae in humans.${ }^{97}$ Negative BOLD changes, when present, are produced with no appreciable spatial pattern, though they are sometimes found near activations. ${ }^{102,105}$ Indeed, the deactivation of resting state networks seen in humans is not seen in animals. The aforementioned differences may reflect evolutionary differences between humans and animals (particularly the resting state network differences) or more likely, differences between the various animal models of epilepsy that have been studied.

In summary, the role of thalamic activation in generalized discharges has been demonstrated in humans and in animal models of epilepsy. In addition, cortical activation is also seen in humans and animals, however, these changes are more focal, suggesting that 'generalized' discharges are perhaps not as generalized over the entire brain as once thought. Widespread, but focal cortical BOLD deactivation associated with GSW is seen in humans in the precuneus, bilateral frontal and temporoparietial cortices as well as the posterior cingulate cortex..$^{67,97}$ These deactivations are localized to areas typically associated with 'default' mode of brain function, suggesting a possible explanation for alteration of awareness during absence seizures in humans.

\section{Pre-ictal state}

Mounting evidence supports the existence of a 'pre-ictal' period during which cerebral activity evolves into a seizure. Evidence supporting the pre-ictal state includes the presence of 


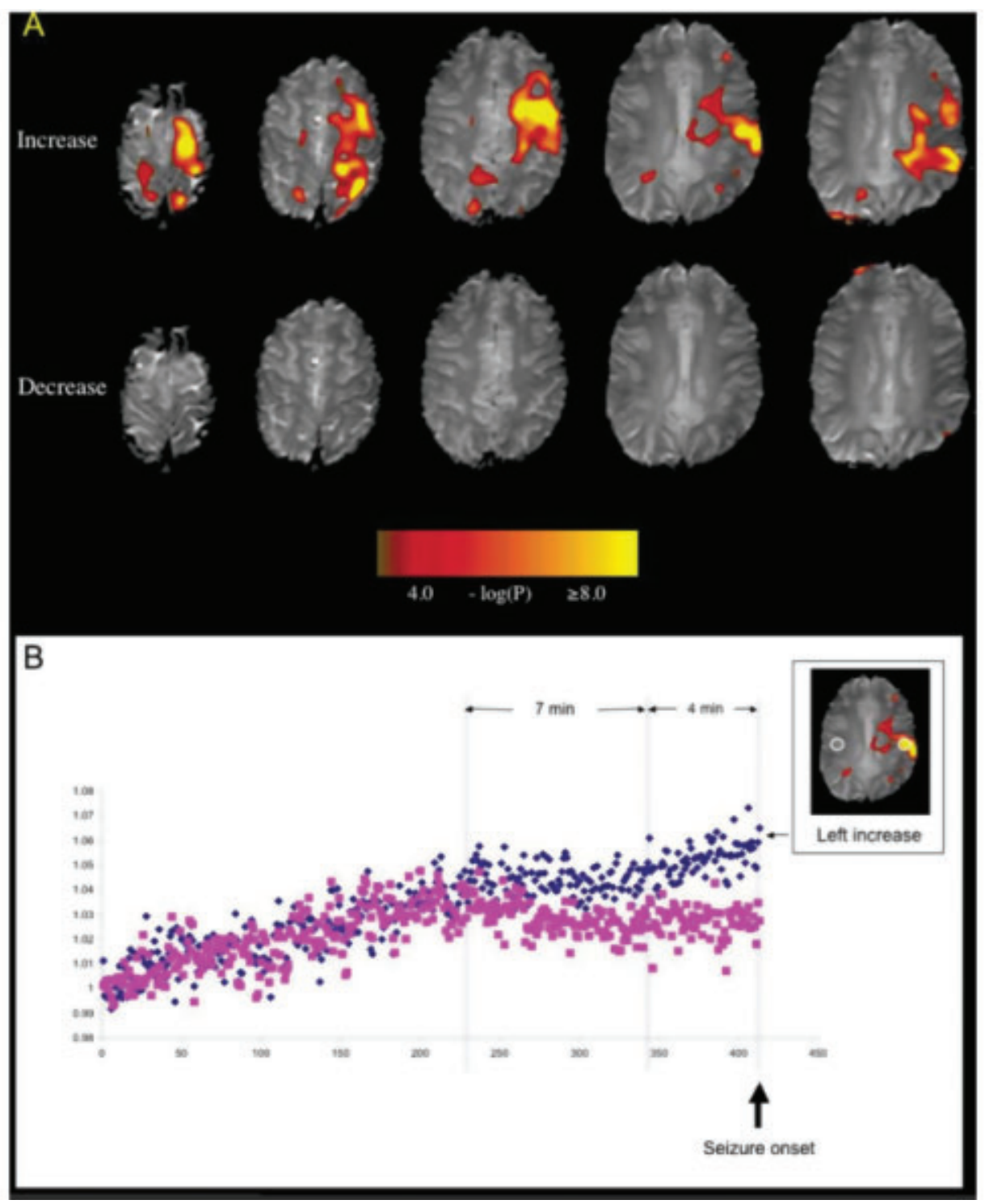

Figure 7: Pre-ictal BOLD signal changes in a patient with refractory seizures. A. Top row. BOLD signal increases were seen in the left frontal lobe and post-central gyrus. Bottom row. No significant BOLD signal decreases were seen. B. The time course of the BOLD signal for 25 minutes prior to seizure. This was measured in a 25 voxel regions of interest (ROI) in the area of maximal BOLD signal (small image). This ROI corresponded to the patient's seizure focus. Increase is shown as diamonds and the BOLD signal in the homologous contralateral region is shown as squares. Image intensity in both regions was normalized to 1 in the first volume (y-axis) and plotted as a function of volume number (one volume every $3.6 \mathrm{~s}$, $x$-axis). Note the signal fluctuates in a similar way in both regions for the first few minutes, followed by a divergence of BOLD signal 11 min prior to the seizure event, with relatively increased signal in the ROI ipsilateral to the seizure focus. This increase is biphasic, with an initial change maintained for $\sim 4 m i n$, followed by a progressive increase until the seizure (vertical lines). Federico et al. Brain 2005;128 (Pt 8):1811-1817.

prodromal symptoms and heart rhythm changes, which may occur minutes to hours prior to seizure onset. ${ }^{107,108}$ In addition, non-linear (chaos) and linear analyses of intracranial EEG recordings have demonstrated EEG changes (not visible on routine inspection of the EEG), which occur several minutes to hours prior to seizure onset. ${ }^{109}$ Furthermore, cerebral blood flow increases have been observed at the seizure focus, beginning several minutes prior to onset of seizure. ${ }^{110,111}$
Two case reports have shown that BOLD signal increases occur one-two minutes prior to clinical or electrographic onset in a patient with Rasmussen's encephalitis ${ }^{8}$ and in a patient with a malignant glioma. ${ }^{112}$ In the only multi-subject study examining pre-ictal BOLD changes to date, signal changes have been shown to occur up to 20 minutes prior to seizure onset. ${ }^{113}$ In the example shown in Figure 7, the BOLD signals measured at the 
seizure focus and in a contralateral homologous region diverge 11 minutes prior to the clinical seizure onset in the absence of detectable EEG changes. ${ }^{113}$ These observations may be related to a recent study of interictal discharges that showed that hemodynamic changes may occur prior to the occurrence of interictal discharges measured using scalp $\mathrm{EEG}^{79}$ (see earlier). Such pre-discharge BOLD signal changes have also been seen previously in two animal studies where the BOLD signal increases occurred either prior to the discharge $e^{80}$ or at the same time (implying hemodynamic changes began several seconds prior). ${ }^{114}$

Thus, the combination of EEG and fMRI has provided the opportunity to investigate where and when pre-ictal brain activation changes occur. Furthermore, an improved characterization of electrophysiological and hemodynamic alterations contributing to ictogenesis may be gained by simultaneously analyzing fMRI and EEG signal changes leading into seizure. This, in turn, may provide future targets for novel treatments or interventions in epilepsy.

\section{Ictal studies}

Performing imaging in an MR scanner immediately prior to and during seizures has appreciable challenges and safety considerations; however, at least seven ictal EEG-fMRI studies have been conducted. $8,76,81,96,97,115$ Only some of these studies will be reviewed.

The first ictal fMRI study was performed by Jackson and colleagues ${ }^{8}$ on a child with Rasmussen's Encephalitis presenting with partial motor seizures. Functional images were collected at ten-second intervals within two ten-minute periods, over which time the subject had five clinical seizures. All seizures were associated with an increase in fMRI signal intensity that started to rise one-two minutes prior to clinical seizure onset.

A case study of a patient with simple partial seizures characterized by psychomotor arrest without falls demonstrated BOLD signal increase at the seizure focus, and decrease in surrounding areas, before a gradual return to baseline. ${ }^{81}$ The authors speculated that BOLD increases might have occurred several seconds prior to distinguishable EEG changes, perhaps indicating pre-ictal changes in BOLD signal. Animal models have confirmed both that ictal BOLD signal changes occur in the seizure focus (as determined by intracranial EEG) ${ }^{63}$ and that BOLD signal changes can occur before appreciable EEG changes in response to epileptiform activity. ${ }^{80}$ Another case study of a patient with late-onset Rasmussen's Encephalitis was performed in which 15 electroclinical events were recorded. ${ }^{115} \mathrm{In}$ this patient, no deactivations were evident and activations were measured in the ipsilateral thalamus and right perisylvian structures (especially in the temporal lobe), which was highly concordant with EEG. ${ }^{115}$ A recent study of 25 brief electrographic seizures in a patient with temporoparietal gray matter nodular heterotopia found BOLD signal changes lasting up to 30 seconds after each three-four second event. ${ }^{116}$ Blood Oxygenation Level Dependent signal increases were seen in the malformation and surrounding cortex. The site of maximal BOLD signal change was in the angular gyrus (i.e., outside the visible malformation), and distant from the site of maximal EEG discharge amplitude, thus raising questions about the precise location of the ictal focus in this patient.
Despite practical challenges, ictal EEG-fMRI recordings have been able to provide information about widespread cortical changes associated with seizures in a manner that is non-invasive and unique from other available methods. The investigation of ictal activity with EEG-fMRI has the potential to provide further insight into the mechanisms underlying the transition into seizures and their subsequent spread.

\section{DISCUSSION}

The continued technical development of simultaneous EEGfMRI has allowed the quality and quantity of information provided by experiments to improve steadily. Removal of ballistocardiographic and gradient switching artifacts allows EEG recorded in the MR environment to reach diagnostic quality. $21,39,41,50$ With the development of strong artifact characterization $^{46,47}$ and artifact reduction methods, ${ }^{21,37,38}$ EEG has been collected in MR scanners with stronger fields to improve the yield of BOLD detection and the accuracy of spatial localization of interictal discharges. Hardware developments have provided further increases in the signal to noise ratio of EEG data. ${ }^{35}$

Refinement of statistical approaches to manage fMRI data has been aimed at improving the detection, strength, and reliability of BOLD responses. Subject motion in clinical investigations remains a formidable challenge but has been addressed using statistical modeling ${ }^{49-51}$ and other more rudimentary approaches such as further securing the subject. It is becoming increasingly evident that the HRF between subjects, and even between different brain regions in the same subject, is not uniform. ${ }^{58,64,77,88}$ Additional questions have been raised about the timing of BOLD changes with respect to measured EEG changes, suggesting that hemodynamic alterations may occur prior to detection of events using EEG. ${ }^{79}$ Investigations of the relationship between BOLD signal changes in different areas of the brain and functional connectivity between so-called 'epilepsy-networks' has raised intriguing questions about the disruption of normal brain function caused by epileptiform activity. ${ }^{97,117,118}$

Concordance between EEG and fMRI localization has not reached $100 \%$ in studies to date, but it is not theoretically expected to reach these values because of the different physiological parameters measured by each method, as well as differences in experimental approaches. Comparison of scalp EEG and functional MRI to intracranial EEG indicates that both approaches correlate well with intracranial EEG in a slightly different manner and consequently provide complementary localizing information, ${ }^{58}$ but further validation against intracranial recordings could improve clinical applicability of EEG-fMRI. It may also be the case that specific frequency bands of EEG correlate better with BOLD response. ${ }^{58}$ Despite the ability to localize functional changes in the entire brain using EEG-fMRI, the precise spatiotemporal mechanisms of epileptiform activity have remained elusive thus far. Investigation of more homogenous patient groups may help resolve this issue. In addition, studying children with identifiable epilepsy syndromes may simplify EEG-fMRI studies as the possible widespread effects of longstanding seizures may be less apparent in this group. ${ }^{92-94}$ 
The EEG-fMRI has great potential to aid in the understanding the pathophysiology of epilepsy. Thus far, insight into the meaning of negative BOLD responses has developed a great deal of interest. Generalized spike-and-wave discharges produce areas of widespread cortical deactivation as measured by fMRI, many of which localize to the 'default', or resting state, mode of the brain..$^{97,98}$ It has been suggested that the suspension of activity in resting state areas may explain the absences often associated with GSW discharges and focal discharges in temporal lobe epilepsy. ${ }^{97,118}$

The EEG-fMRI studies have implicated distant cortical and subcortical structures in seemingly 'focal' epilepsy; ${ }^{66,75}$ however, the precise role of these sites has not been fully explained. Understanding the role of these sites would be particularly useful in a clinical setting to determine suitability for surgery and for identifying surgical targets as already demonstrated in a previous study. ${ }^{89}$ Despite this, current clinical application of EEG-fMRI has been limited and requires further validation. Of note, fMRI (alone or in conjunction with EEG) is being increasingly used in a clinical setting to replace sodium amytal testing and to determine language hemispheric dominance, [ ${ }^{119,120}$ for review], although further refinements of these procedures may be necessary. ${ }^{121}$

\section{CONCLUSION}

The information provided by EEG-fMRI is unique as it measures changes in brain activity/state associated with epileptiform activity at a high spatial resolution, providing whole brain coverage and no radiation risk to the patient. The EEGfMRI studies have already yielded some insights into the pathophysiology of epilepsy (i.e., default state, pre-ictal state), which can be expanded further with a more complete understanding of what each modality measures. Currently, EEG-fMRI is used by some centers in a clinical setting to complement other clinical investigations such as video-EEG monitoring, PET, SPECT, structural MRI, or neuropsychological testing. With further understanding of the relationship of the BOLD signal to interictal and ictal discharges, it is expected that EEG-fMRI will be used more widely in a clinical setting.

\section{ACKNOWLEDGEMENTS}

The authors thank Elizabeth Jensen and Daniel Pittman for help in preparing the manuscript and figures. Paolo Federico is funded by Canadian Institutes of Health Research and Alberta Heritage Foundation for Medical Research. Cameron JB Cunningham has received studentships from the Savoy Foundation for Epilepsy and the Canadian Institutes of Health Research.

\section{REFERENCES}

1. Gibbs FA, Lennox WG, Gibbs EL. Cerebral blood flow preceding and accompanying epileptic seizures in man. Arch Neurol Psychiatry. 1934;(32):257-72.

2. Collins RC, Caston TV. Functional anatomy of occipital lobe seizures: an experimental study in rats. Neurology. 1979 May;29(5):705-16.

3. Collins RC. Use of cortical circuits during focal penicillin seizures: an autoradiographic study with [14C]deoxyglucose. Brain Res. 1978 Jul 21;150(3):487-501.
4. Van Paesschen W, Dupont P, Sunaert S, Goffin K, Van Laere K. The use of SPECT and PET in routine clinical practice in epilepsy. Curr Opin Neurol. 2007 Apr;20(2):194-202.

5. Rowe CC, Berkovic SF, Austin MC, Saling M, Kalnins RM, McKay WJ, et al. Visual and quantitative analysis of interictal SPECT with technetium-99m-HMPAO in temporal lobe epilepsy. J Nucl Med. 1991 Sep;32(9):1688-94.

6. Spencer SS. The relative contributions of MRI, SPECT, and PET imaging in epilepsy. Epilepsia. 1994;35 Suppl 6:S72-89.

7. Devous MD, Sr., Thisted RA, Morgan GF, Leroy RF, Rowe CC. SPECT brain imaging in epilepsy: a meta-analysis. J Nucl Med. 1998 Feb;39(2):285-93.

8. Jackson GD, Connelly A, Cross JH, Gordon I, Gadian DG. Functional magnetic resonance imaging of focal seizures. Neurology. 1994 May;44(5):850-6.

9. Warach S, Ives JR, Schlaug G, Patel MR, Darby DG, Thangaraj V, et al. EEG-triggered echo-planar functional MRI in epilepsy. Neurology. 1996 Jul;47(1):89-93.

10. Seeck M, Lazeyras F, Michel CM, Blanke O, Gericke CA, Ives J, et al. Non-invasive epileptic focus localization using EEGtriggered functional MRI and electromagnetic tomography. Electroencephalogr Clin Neurophysiol. 1998 Jun;106(6):508-12.

11. Krakow K, Woermann FG, Symms MR, Allen PJ, Lemieux L, Barker GJ, et al. EEG-triggered functional MRI of interictal epileptiform activity in patients with partial seizures. Brain. 1999 Sep;122 (Pt 9):1679-88.

12. Ogawa S, Tank DW, Menon R, Ellermann JM, Kim SG, Merkle H, et al. Intrinsic signal changes accompanying sensory stimulation: functional brain mapping with magnetic resonance imaging. Proc Natl Acad Sci U S A. 1992 Jul 1;89(13):5951-5.

13. Logothetis NK, Pauls J, Augath M, Trinath T, Oeltermann A. Neurophysiological investigation of the basis of the fMRI signal. Nature. 2001 Jul 12;412(6843):150-7.

14. Logothetis NK. The underpinnings of the BOLD functional magnetic resonance imaging signal. J Neurosci. 2003 May 15;23(10):3963-71.

15. Menon RS. Imaging function in the working brain with fMRI. Curr Opin Neurobiol. 2001 Oct;11(5):630-6.

16. Yacoub E, Hu X. Detection of the early decrease in fMRI signal in the motor area. Magn Reson Med. 2001 Feb;45(2):184-90.

17. Yacoub E, Le TH, Ugurbil K, Hu X. Further evaluation of the initial negative response in functional magnetic resonance imaging. Magn Reson Med. 1999 Mar;41(3):436-41.

18. Malonek D, Grinvald A. Interactions between electrical activity and cortical microcirculation revealed by imaging spectroscopy: implications for functional brain mapping. Science. $1996 \mathrm{Apr}$ 26;272(5261):551-4.

19. Yacoub E, Ugurbil K, Harel N. The spatial dependence of the poststimulus undershoot as revealed by high-resolution BOLDand CBV-weighted fMRI. J Cereb Blood Flow Metab. 2006 May;26(5):634-44

20. Ives JR, Warach S, Schmitt F, Edelman RR, Schomer DL. Monitoring the patient's EEG during echo planar MRI. Electroencephalogr Clin Neurophysiol. 1993 Dec;87(6):417-20.

21. Allen PJ, Josephs O, Turner R. A method for removing imaging artifact from continuous EEG recorded during functional MRI. Neuroimage. 2000 Aug;12(2):230-9.

22. Kaufmann C, Wehrle R, Wetter TC, Holsboer F, Auer DP, Pollmacher T, et al. Brain activation and hypothalamic functional connectivity during human non-rapid eye movement sleep: an EEG/fMRI study. Brain. 2006 Mar;129(Pt 3):655-67.

23. Krakow K, Lemieux L, Messina D, Scott CA, Symms MR, Duncan JS, et al. Spatio-temporal imaging of focal interictal epileptiform activity using EEG-triggered functional MRI. Epileptic Disord. 2001 Jun;3(2):67-74.

24. Lazeyras F, Blanke O, Perrig S, Zimine I, Golay X, Delavelle J, et al. EEG-triggered functional MRI in patients with pharmacoresistant epilepsy. J Magn Reson Imaging. 2000 Jul; 12(1):177-85.

25. Schomer DL, Bonmassar G, Lazeyras F, Seeck M, Blum A, Anami $\mathrm{K}$, et al. EEG-Linked functional magnetic resonance imaging in epilepsy and cognitive neurophysiology. J Clin Neurophysiol. 2000 Jan; $17(1): 43-58$ 
26. Blumenfeld H. Functional MRI studies of animal models in epilepsy. Epilepsia. 2007;48 Suppl 4:18-26.

27. Mirsattari SM, Ives JR, Leung LS, Menon RS. EEG monitoring during functional MRI in animal models. Epilepsia. 2007;48 Suppl 4:37-46.

28. Feychting M. Health effects of static magnetic fields--a review of the epidemiological evidence. Prog Biophys Mol Biol. 2005 Feb-Apr;87(2-3):241-6

29. Sienkiewicz Z. Rapporteur report: implications for exposure guidelines. Prog Biophys Mol Biol. 2005 Feb-Apr;87(2-3): 365-72.

30. Medical magnetic resonance (MR) procedures: protection of patients. Health Phys. 2004 Aug;87(2):197-216.

31. Silva AK, Silva EL, Egito ES, Carrico AS. Safety concerns related to magnetic field exposure. Radiat Environ Biophys. 2006 Nov;45(4):245-52.

32. Lemieux L, Allen PJ, Franconi F, Symms MR, Fish DR. Recording of EEG during fMRI experiments: patient safety. Magn Reson Med. 1997 Dec;38(6):943-52.

33. Yeung CJ, Susil RC, Atalar E. RF safety of wires in interventional MRI: using a safety index. Magn Reson Med. 2002 Jan;47(1):187-93.

34. Angelone LM, Potthast A, Segonne F, Iwaki S, Belliveau JW, Bonmassar G. Metallic electrodes and leads in simultaneous EEG-MRI: specific absorption rate (SAR) simulation studies. Bioelectromagnetics. 2004 May;25(4):285-95.

35. Vasios CE, Angelone LM, Purdon PL, Ahveninen J, Belliveau JW, Bonmassar G. EEG/(f)MRI measurements at 7 Tesla using a new EEG cap ("InkCap"). Neuroimage. 2006 Dec;33(4):1082-92.

36. Al-Asmi A, Benar CG, Gross DW, Khani YA, Andermann F, Pike $\mathrm{B}$, et al. fMRI activation in continuous and spike-triggered EEGfMRI studies of epileptic spikes. Epilepsia. 2003 Oct:44(10):1328-39.

37. Hoffmann A, Jager L, Werhahn KJ, Jaschke M, Noachtar S, Reiser M. Electroencephalography during functional echo-planar imaging: detection of epileptic spikes using post-processing methods. Magn Reson Med. 2000 Nov;44(5):791-8.

38. Goldman RI, Stern JM, Engel J, Jr., Cohen MS. Acquiring simultaneous EEG and functional MRI. Clin Neurophysiol. 2000 Nov;111(11):1974-80.

39. Mirsattari SM, Ives JR, Bihari F, Leung LS, Menon RS, Bartha R. Real-time display of artifact-free electroencephalography during functional magnetic resonance imaging and magnetic resonance spectroscopy in an animal model of epilepsy. Magn Reson Med. $2005 \mathrm{Feb} ; 53(2): 456-64$

40. Salek-Haddadi A, Diehl B, Hamandi K, Merschhemke M, Liston A, Friston $\mathrm{K}$, et al. Hemodynamic correlates of epileptiform discharges: an EEG-fMRI study of 63 patients with focal epilepsy. Brain Res. 2006 May 9;1088(1):148-66.

41. Allen PJ, Polizzi G, Krakow K, Fish DR, Lemieux L. Identification of EEG events in the MR scanner: the problem of pulse artifact and a method for its subtraction. Neuroimage. 1998 Oct; $8(3): 229-39$

42. Lagerlund TD, Sharbrough FW, Busacker NE. Spatial filtering of multichannel electroencephalographic recordings through principal component analysis by singular value decomposition. $\mathbf{J}$ Clin Neurophysiol. 1997 Jan; 14(1):73-82.

43. Srivastava G, Crottaz-Herbette S, Lau KM, Glover GH, Menon V. ICA-based procedures for removing ballistocardiogram artifacts from EEG data acquired in the MRI scanner. Neuroimage. 2005 Jan 1;24(1):50-60

44. Mantini D, Perrucci MG, Cugini S, Ferretti A, Romani GL, Del Gratta C. Complete artifact removal for EEG recorded during continuous fMRI using independent component analysis. Neuroimage. 2007 Jan 15;34(2):598-607.

45. Nakamura W, Anami K, Mori T, Saitoh O, Cichocki A, Amari S. Removal of ballistocardiogram artifacts from simultaneously recorded EEG and fMRI data using independent component analysis. IEEE Trans Biomed Eng. 2006 Jul:53(7):1294-308.

46. Masterton RA, Abbott DF, Fleming SW, Jackson GD. Measurement and reduction of motion and ballistocardiogram artefacts from simultaneous EEG and fMRI recordings. Neuroimage. 2007 Aug $1 ; 37(1): 202-11$.
47. Mandelkow H, Halder P, Boesiger P, Brandeis D. Synchronization facilitates removal of MRI artefacts from concurrent EEG recordings and increases usable bandwidth. Neuroimage. 2006 Sep;32(3):1120-6.

48. Kobayashi E, Bagshaw AP, Grova C, Dubeau F, Gotman J. Negative BOLD responses to epileptic spikes. Hum Brain Mapp. 2006 Jun;27(6):488-97.

49. Friston KJ, Williams S, Howard R, Frackowiak RS, Turner R. Movement-related effects in fMRI time-series. Magn Reson Med. 1996 Mar;35(3):346-55.

50. Salek-Haddadi A, Lemieux L, Merschhemke M, Diehl B, Allen PJ, Fish DR. EEG quality during simultaneous functional MRI of interictal epileptiform discharges. Magn Reson Imaging. 2003 Dec;21(10):1159-66.

51. Lemieux L, Salek-Haddadi A, Lund TE, Laufs H, Carmichael D. Modelling large motion events in fMRI studies of patients with epilepsy. Magn Reson Imaging. 2007 July;25(6):894-901

52. Smith AM, Lewis BK, Ruttimann UE, Ye FQ, Sinnwell TM, Yang $\mathrm{Y}$, et al. Investigation of low frequency drift in fMRI signal. Neuroimage. 1999 May;9(5):526-33.

53. Benar CG, Gross DW, Wang Y, Petre V, Pike B, Dubeau F, et al. The BOLD response to interictal epileptiform discharges. Neuroimage. 2002 Nov;17(3):1182-92.

54. Huang CC, Liou, M., Cheng, P.E., editor. Baseline correction of functional MR time courses with PCA. Proceedings of the 9th International Conference on Neural Information Processing; 2002 18-22 Nov. 2002.

55. Foerster BU, Tomasi D, Caparelli EC. Magnetic field shift due to mechanical vibration in functional magnetic resonance imaging. Magn Reson Med. 2005 Nov;54(5):1261-7.

56. Mirsattari SM, Wang Z, Ives JR, Bihari F, Leung LS, Bartha R, et al. Linear aspects of transformation from interictal epileptic discharges to BOLD fMRI signals in an animal model of occipital epilepsy. Neuroimage. 2006 May 1;30(4):1133-48.

57. Bagshaw AP, Kobayashi E, Dubeau F, Pike GB, Gotman J. Correspondence between EEG-fMRI and EEG dipole localisation of interictal discharges in focal epilepsy. Neuroimage. 2006 Apr 1;30(2):417-25.

58. Benar CG, Grova C, Kobayashi E, Bagshaw AP, Aghakhani Y, Dubeau F, et al. EEG-fMRI of epileptic spikes: concordance with EEG source localization and intracranial EEG. Neuroimage. 2006 May 1;30(4):1161-70.

59. Patel MR, Blum A, Pearlman JD, Yousuf N, Ives JR, Saeteng S, et al. Echo-planar functional MR imaging of epilepsy with concurrent EEG monitoring. AJNR Am J Neuroradiol. 1999 Nov-Dec;20(10):1916-9.

60. Archer JS, Briellman RS, Abbott DF, Syngeniotis A, Wellard RM, Jackson GD. Benign epilepsy with centro-temporal spikes: spike triggered fMRI shows somato-sensory cortex activity. Epilepsia. 2003 Feb;44(2):200-4

61. Archer JS, Briellmann RS, Syngeniotis A, Abbott DF, Jackson GD. Spike-triggered fMRI in reading epilepsy: involvement of left frontal cortex working memory area. Neurology. 2003 Feb $11 ; 60(3): 415-21$

62. Jager L, Werhahn KJ, Hoffmann A, Berthold S, Scholz V, Weber J, et al. Focal epileptiform activity in the brain: detection with spike-related functional MR imaging--preliminary results. Radiology. 2002 Jun;223(3):860-9.

63. Opdam HI, Federico P, Jackson GD, Buchanan J, Abbott DF, Fabinyi GC, et al. A sheep model for the study of focal epilepsy with concurrent intracranial EEG and functional MRI. Epilepsia. 2002 Aug;43(8):779-87.

64. Bagshaw AP, Aghakhani Y, Benar CG, Kobayashi E, Hawco C, Dubeau F, et al. EEG-fMRI of focal epileptic spikes: analysis with multiple haemodynamic functions and comparison with gadolinium-enhanced MR angiograms. Hum Brain Mapp. 2004 Jul;22(3):179-92.

65. Aghakhani Y, Kobayashi E, Bagshaw AP, Hawco C, Benar CG, Dubeau $\mathrm{F}$, et al. Cortical and thalamic fMRI responses in partial epilepsy with focal and bilateral synchronous spikes. Clin Neurophysiol. 2006 Jan;117(1):177-91. 
66. Federico P, Archer JS, Abbott DF, Jackson GD. Cortical/subcortical BOLD changes associated with epileptic discharges: an EEGfMRI study at 3 T. Neurology. 2005 Apr 12;64(7):1125-30.

67. Archer JS, Abbott DF, Waites AB, Jackson GD. fMRI "deactivation" of the posterior cingulate during generalized spike and wave. Neuroimage. 2003 Dec;20(4):1915-22.

68. Gusnard DA, Raichle ME, Raichle ME. Searching for a baseline: functional imaging and the resting human brain. Nat Rev Neurosci. 2001 Oct;2(10):685-94.

69. Shmuel A, Yacoub E, Pfeuffer J, Van de Moortele PF, Adriany G, $\mathrm{Hu} \mathrm{X}$, et al. Sustained negative BOLD, blood flow and oxygen consumption response and its coupling to the positive response in the human brain. Neuron. 2002 Dec 19;36(6):1195-210.

70. Stefanovic B, Warnking JM, Pike GB. Hemodynamic and metabolic responses to neuronal inhibition. Neuroimage. 2004 Jun;22(2):771-8.

71. Holmes GL, Sarkisian M, Ben-Ari Y, Liu Z, Chevassus-Au-Louis N. Consequences of cortical dysplasia during development in rats. Epilepsia. 1999 May;40(5):537-44.

72. Zilles K, Qu M, Schleicher A, Luhmann HJ. Characterization of neuronal migration disorders in neocortical structures: quantitative receptor autoradiography of ionotropic glutamate, GABA(A) and GABA(B) receptors. Eur J Neurosci. 1998 Oct;10(10):3095-106.

73. Silva-Barrat C, Menini C, Bryere P, Naquet R. Multiunitary activity analysis of cortical and subcortical structures in paroxysmal discharges and grand mal seizures in photosensitive baboons. Electroencephalogr Clin Neurophysiol. 1986 Nov;64(5):455-68.

74. Morillo LE, Ebner TJ, Bloedel JR. The early involvement of subcortical structures during the development of a cortical seizure focus. Epilepsia. 1982 Dec;23(6):571-85.

75. Kobayashi E, Bagshaw AP, Benar CG, Aghakhani Y, Andermann F, Dubeau $\mathrm{F}$, et al. Temporal and extratemporal BOLD responses to temporal lobe interictal spikes. Epilepsia. 2006 Feb;47(2): 343-54.

76. Aghakhani Y, Bagshaw AP, Benar CG, Hawco C, Andermann F, Dubeau $F$, et al. fMRI activation during spike and wave discharges in idiopathic generalized epilepsy. Brain. 2004 May; 127(Pt 5):1127-44

77. Kang JK, Benar C, Al-Asmi A, Khani YA, Pike GB, Dubeau F, et al. Using patient-specific hemodynamic response functions in combined EEG-fMRI studies in epilepsy. Neuroimage. 2003 Oct;20(2):1162-70.

78. Lu Y, Bagshaw AP, Grova C, Kobayashi E, Dubeau F, Gotman J. Using voxel-specific hemodynamic response function in EEGfMRI data analysis. Neuroimage. 2006 Aug 1;32(1):238-47.

79. Hawco CS, Bagshaw AP, Lu Y, Dubeau F, Gotman J. BOLD changes occur prior to epileptic spikes seen on scalp EEG. Neuroimage. 2007 May 1;35(4):1450-8.

80. Makiranta M, Ruohonen J, Suominen K, Niinimaki J, Sonkajarvi E, Kiviniemi V, et al. BOLD signal increase preceeds EEG spike activity--a dynamic penicillin induced focal epilepsy in deep anesthesia. Neuroimage. 2005 Oct 1;27(4):715-24.

81. Salek-Haddadi A, Merschhemke M, Lemieux L, Fish DR. Simultaneous EEG-Correlated Ictal fMRI. Neuroimage. 2002 May;16(1):32-40.

82. Lemieux L, Salek-Haddadi A, Josephs O, Allen P, Toms N, Scott C, et al. Event-related fMRI with simultaneous and continuous EEG: description of the method and initial case report. Neuroimage. 2001 Sep;14(3):780-7.

83. Nunez PL, Silberstein RB. On the relationship of synaptic activity to macroscopic measurements: does co-registration of EEG with fMRI make sense? Brain Topogr. 2000 Winter;13(2):79-96.

84. Tao JX, Ray A, Hawes-Ebersole S, Ebersole JS. Intracranial EEG substrates of scalp EEG interictal spikes. Epilepsia. 2005 May;46(5):669-76.

85. Ebersole JS. Defining epileptogenic foci: past, present, future. J Clin Neurophysiol. 1997 Nov;14(6):470-83.

86. Ebersole JS, Pacia SV. Localization of temporal lobe foci by ictal EEG patterns. Epilepsia. 1996 Apr;37(4):386-99.

87. Luders HO, Engel Jr., J, Munari, C. Surgical treatment of the epilepsies. New York: Raven Press Ltd; 1993.
88. Lu Y, Grova C, Kobayashi E, Dubeau F, Gotman J. Using voxelspecific hemodynamic response function in EEG-fMRI data analysis: an estimation and detection model. Neuroimage. 2007 Jan 1;34(1):195-203

89. Zijlmans M, Huiskamp G, Hersevoort M, Seppenwoolde JH, van Huffelen AC, Leijten FS. EEG-fMRI in the preoperative workup for epilepsy surgery. Brain. $2007 \mathrm{Sep} ; 130(\mathrm{Pt}$ 9):2343-53.

90. Hamandi K, Salek-Haddadi A, Fish DR, Lemieux L. EEG/ functional MRI in epilepsy: The queen square experience. J Clin Neurophysiol. 2004 Jul-Aug;21(4):241-8.

91. Gotman J, Kobayashi E, Bagshaw AP, Benar CG, Dubeau F. Combining EEG and fMRI: a multimodal tool for epilepsy research. J Magn Reson Imaging. 2006 Jun;23(6):906-20.

92. Boor S, Vucurevic G, Pfleiderer C, Stoeter P, Kutschke G, Boor R. EEG-related functional MRI in benign childhood epilepsy with centrotemporal spikes. Epilepsia. 2003 May;44(5):688-92.

93. Leal A, Dias A, Vieira JP, Secca M, Jordao C. The BOLD effect of interictal spike activity in childhood occipital lobe epilepsy. Epilepsia. 2006 Sep;47(9):1536-42.

94. De Tiege X, Laufs H, Boyd SG, Harkness W, Allen PJ, Clark CA, et al. EEG-fMRI in children with pharmacoresistant focal epilepsy. Epilepsia. 2007 Feb;48(2):385-9.

95. Hill RA, Chiappa KH, Huang-Hellinger F, Jenkins BG. Hemodynamic and metabolic aspects of photosensitive epilepsy revealed by functional magnetic resonance imaging and magnetic resonance spectroscopy. Epilepsia. 1999 Jul;40(7): 912-20.

96. Salek-Haddadi A, Lemieux L, Merschhemke M, Friston KJ, Duncan JS, Fish DR. Functional magnetic resonance imaging of human absence seizures. Ann Neurol. 2003 May;53(5):663-7.

97. Gotman J, Grova C, Bagshaw A, Kobayashi E, Aghakhani Y, Dubeau F. Generalized epileptic discharges show thalamocortical activation and suspension of the default state of the brain. Proc Natl Acad Sci USA. 2005 Oct 18;102(42): 15236-40.

98. Raichle ME, MacLeod AM, Snyder AZ, Powers WJ, Gusnard DA, Shulman GL. A default mode of brain function. Proc Natl Acad Sci U S A. 2001 Jan 16;98(2):676-82.

99. Hamandi K, Laufs H, Noth U, Carmichael DW, Duncan JS, Lemieux L. BOLD and perfusion changes during epileptic generalised spike wave activity. Neuroimage. 2008 Jan 15; 39(2):608-18

100. Laufs H, Hamandi K, Salek-Haddadi A, Kleinschmidt AK, Duncan JS, Lemieux L. Temporal lobe interictal epileptic discharges affect cerebral activity in "default mode" brain regions. Hum Brain Mapp. 2007 Oct;28(10):1023-32.

101. Tenney JR, Marshall PC, King JA, Ferris CF. fMRI of generalized absence status epilepticus in conscious marmoset monkeys reveals corticothalamic activation. Epilepsia. 2004 Oct;45(10): 1240-7.

102. Tenney JR, Duong TQ, King JA, Ludwig R, Ferris CF. Corticothalamic modulation during absence seizures in rats: a functional MRI assessment. Epilepsia. 2003 Sep;44(9):1133-40.

103. Van Camp N, D'Hooge R, Verhoye M, Peeters RR, De Deyn PP, Van der Linden A. Simultaneous electroencephalographic recording and functional magnetic resonance imaging during pentylenetetrazol-induced seizures in rat. Neuroimage. $2003 \mathrm{Jul}$; 19(3):627-36.

104. Brevard ME, Kulkarni P, King JA, Ferris CF. Imaging the neural substrates involved in the genesis of pentylenetetrazol-induced seizures. Epilepsia. 2006 Apr;47(4):745-54.

105. Tenney JR, Duong TQ, King JA, Ferris CF. FMRI of brain activation in a genetic rat model of absence seizures. Epilepsia. 2004 Jun;45(6):576-82.

106. Nersesyan H, Hyder F, Rothman DL, Blumenfeld H. Dynamic fMRI and EEG recordings during spike-wave seizures and generalized tonic-clonic seizures in WAG/Rij rats. J Cereb Blood Flow Metab. 2004 Jun;24(6):589-99.

107. Delamont RS, Julu PO, Jamal GA. Changes in a measure of cardiac vagal activity before and after epileptic seizures. Epilepsy Res. 1999 Jun;35(2):87-94. 
108. Boylan LS. Peri-ictal behavioral and cognitive changes. Epilepsy Behav. 2002 Feb;3(1):16-26.

109. Litt B, Esteller R, Echauz J, D'Alessandro M, Shor R, Henry T, et al. Epileptic seizures may begin hours in advance of clinical onset: a report of five patients. Neuron. 2001 Apr;30(1):51-64.

110. Weinand ME, Carter LP, el-Saadany WF, Sioutos PJ, Labiner DM, Oommen KJ. Cerebral blood flow and temporal lobe epileptogenicity. J Neurosurg. 1997 Feb;86(2):226-32.

111. Baumgartner C, Serles W, Leutmezer F, Pataraia E, Aull S, Czech T, et al. Preictal SPECT in temporal lobe epilepsy: regional cerebral blood flow is increased prior to electroencephalography-seizure onset. J Nucl Med. 1998 Jun;39(6): 978-82.

112. Krings T, Topper R, Reinges MH, Foltys H, Spetzger U, Chiappa $\mathrm{KH}$, et al. Hemodynamic changes in simple partial epilepsy: a functional MRI study. Neurology. 2000 Jan 25;54(2):524-7.

113. Federico P, Abbott DF, Briellmann RS, Harvey AS, Jackson GD. Functional MRI of the pre-ictal state. Brain. 2005 Aug;128(Pt 8):1811-7.

114. Keogh BP, Cordes D, Stanberry L, Figler BD, Robbins CA, Tempel BL, et al. BOLD-fMRI of PTZ-induced seizures in rats. Epilepsy Res. 2005 Aug-Sep;66(1-3):75-90.

115. Di Bonaventura C, Carnfi M, Vaudano AE, Pantano P, Garreffa G, Le Piane $\mathrm{E}$, et al. Ictal hemodynamic changes in late-onset rasmussen encephalitis. Ann Neurol. 2006 Feb;59(2):432-3.
116. Kobayashi E, Hawco CS, Grova C, Dubeau F, Gotman J. Widespread and intense BOLD changes during brief focal electrographic seizures. Neurology. 2006 Apr 11;66(7):1049-55.

117. Waites AB, Briellmann RS, Saling MM, Abbott DF, Jackson GD. Functional connectivity networks are disrupted in left temporal lobe epilepsy. Ann Neurol. 2006 Feb;59(2):335-43.

118. Laufs H, Lengler U, Hamandi K, Kleinschmidt A, Krakow K. Linking generalized spike-and-wave discharges and resting state brain activity by using EEG/fMRI in a patient with absence seizures. Epilepsia. 2006 Feb;47(2):444-8.

119. Pelletier I, Sauerwein HC, Lepore F, Saint-Amour D, Lassonde M. Non-invasive alternatives to the Wada test in the presurgical evaluation of language and memory functions in epilepsy patients. Epileptic Disord. 2007 Jun;9(2):111-26.

120. Abou-Khalil B. An update on determination of language dominance in screening for epilepsy surgery: the Wada test and newer noninvasive alternatives. Epilepsia. 2007 Mar;48(3):442-55.

121. Benke T, Koylu B, Visani P, Karner E, Brenneis C, Bartha L, et al. Language lateralization in temporal lobe epilepsy: a comparison between fMRI and the Wada test. Epilepsia. 2006 Aug;47(8): 1308-19. 